Pacific Journal of Mathematics

CONGRUENCE RELATIONS AND MULTIPLICITY TYPES OF thomas Paul Whale y 


\title{
CONGRUENCE RELATIONS AND MULTIPLICITY TYPES OF ALGEBRAS
}

\author{
B. Jónsson AND T. P. Whaley
}

\begin{abstract}
Given two multiplicity types $\mu$ and $\mu^{\prime}$, the following two conditions are shown to be equivalent: (1) For every algebra $A$ of multiplicity type $\mu$ there exists an algebra $A^{\prime}$ of multiplicity type $\mu^{\prime}$ such that $A$ and $A^{\prime}$ have exactly the same congruence relations. (2) For every $k>0, \mu_{k}+\mu_{k+1}+\cdots \leqq \mu_{k}^{\prime}+$ $\mu_{k+1}^{\prime}+\cdots$.
\end{abstract}

Introduction. Given an algebra $A=\left\langle U, f_{i}(i \in I)\right\rangle$, we let Con $(A)$ be the lattice of congruence relations over $A$. By the multiplicity type of $A$ we mean the sequence $\mu=\left\langle\mu_{0}, \mu_{1}, \cdots, \mu_{n}, \cdots\right\rangle$ where $\mu_{n}$ is the number of indices $i$ for which the rank of $f_{i}$ is $n$. The purpose of this paper is to prove the following result:

THEOREM. If $\mu$ and $\mu^{\prime}$ are multiplicity types with the property that for every algebra $A$ of the multiplicity type $\mu$ there exists an algebra $A^{\prime}$ of the multiplicity type $\mu^{\prime}$ such that $\operatorname{Con}(A)=\operatorname{Con}\left(A^{\prime}\right)$, then $\mu_{k}+\mu_{k+1}+\cdots \leqq \mu_{k}^{\prime}+\mu_{k+1}^{\prime}+\cdots$ for $k=1,2, \cdots$.

This result was conjectured in [3], and the special case $k=1$ was proved in [5]. The theorem was announced in [4] for the case when the multiplicity types are finite, and in [2] without that restriction.

The converse of our theorem is also true; its proof easily reduces to an elementary but somewhat tedious set-theoretic argument. Roughly, we want to show that if the given inequalities hold, then any algebra of the multiplicity type $\mu$ can be transformed into an algebra of the multiplicity type $\mu^{\prime}$ by adding dummy arguments to some of the operations and by introducing new operations that do not affect the congruence relations. (Since operations of rank zero do not affect the congruence relations, we may assume that $\mu_{0}=\mu_{0}^{\prime}=0$.) It is clearly sufficient to show that, given two sets $I$ and $I^{\prime}$, partitioned into subsets $I_{n}$ and $I_{n}^{\prime}$, respectively, with $\left|I_{n}\right|=\mu_{n}$ and $\left|I_{n}^{\prime}\right|=\mu_{n}^{\prime}$ $(n=1,2, \cdots)$, there exists a one-to-one map $\phi: I \rightarrow I^{\prime}$ such that, for each $i \in I_{n}, \phi(i)$ belongs to some $I_{m}^{\prime}$ with $m \geqq n$. The existence of such a map will certainly be assured if we show that $I^{\prime}$ can be partitioned into subsets $J_{n}$ with $\mu_{n} \leqq\left|J_{n}\right|$ for $n=1,2, \cdots$ in such a way that each member of $J_{n}$ belongs to some $I_{m}^{\prime}$ with $m \geqq n$. We shall in fact construct a double sequence of $\operatorname{sets} J_{p, n}(p \geqq 0, n \geqq 1)$ with $J_{0, n}=I_{n}^{\prime}$ in such a way that

(a) for a fixed $p$, the sets $J_{p, n}$ form a partitioning of $I^{\prime}$, 
(b) $J_{p+1, k}=J_{p, k}$ for $k=1,2, \cdots, p$.

(c) each member of $J_{p, n}$ belongs to some $J_{p+1, k}$ with $k \leqq n$

(d) $\mu_{n} \leqq\left|J_{n, n}\right|$ for $n=1,2, \cdots$.

The sets $J_{n}=J_{n, n}$ are then easily seen to form the required partitioning of $I^{\prime}$.

The sets $J_{p, n}$ are defined successively for $p=1,2, \cdots$, and for the purpose of the recursive definition we require the cardinals $\mu_{p, n}=$ $\left|J_{p, n}\right|$ to satisfy the further condition that

(e) $\mu_{k}+\mu_{k+1}+\cdots \leqq \mu_{p, k}+\mu_{p, k+1}+\cdots$ for $k=1,2, \cdots$.

This condition is satisfied by hypothesis for $p=0$. Assuming that the sets $J_{p, n}$ have been defined for a given value of $p$, we indicate how the sets $J_{p+1, n}$ are to be chosen, but leave it to the reader to verify that the conditions (a)-(e) are satisfied.

We let $J_{p+1, n}=J_{p, n}$ for $n=1,2, \cdots, p$, but consider three cases in defining the sets $J_{p+1, n}$ with $n>p$.

Case 1. $\mu_{p, n}$ is infinite for some $n>p$.

For $n>p+1$ let $J_{p+1, n}=J_{p, n}$ if $\mu_{p, n}$ is finite, but if $\mu_{p, n}$ is infinite, partition $J_{p, n}$ into two sets, $J_{p+1, n}$ and $K_{p, n}$, both of cardinality $\mu_{p, n}$. Let $J_{p+1, p+1}$ be the union of $J_{p, p+1}$ and of the sets $K_{p, n}$.

Case 2. $\mu_{p, n}$ is finite for all $n>p$, but $\mu_{p, n} \neq 0$ for infinitely many values of $n$.

In this case the set $J_{p, p+2} \cup J_{p, p+3} \cup \cdots$ is countably infinite, and its members can be arranged into a sequence without repetitions, say $a_{1}, a_{2}, a_{3}, \cdots$, in such a way that the members of $J_{p, n}$ always precede the members of $J_{p, n+1}$. Let $J_{p+1, p+1}$ consist of the elements of $J_{p, p+1}$ together with the odd numbered terms of this sequence. Let $J_{p+1, p+2}$ be the set consisting of the first $\mu_{p, p+2}$ even numbered terms of this sequence, $J_{p+1, p+3}$ the set consisting of the next $\mu_{p, p+3}$ even numbered terms, etc.

Case 3. $\mu_{p, n}$ is finite for all $n>p$ and equals zero for all but finitely many $n>p$.

Choose $m$ so that $\mu_{p, n}=0$ for $n>p+m$. Then $\mu_{n}=0$ for $n>$ $p+m$. The elements of the set $J_{p, p+1} \cup J_{p, p+2} \cup \cdots \cup J_{p, p+m}$ can be arranged into a finite sequence without repetitions, say $a_{1}, a_{2}, \cdots, a_{q}$, in such a way that the members of $J_{p, p+n}$ always precede the members of $J_{p, p+n-1}$. Let $J_{p+1, p+n}=\varnothing$ for $n>m$, let $J_{p+1, p+m}$ be the set consisting of the first $\mu_{p+m}$ terms of the sequence, $J_{p+1, p+m-1}$ the set consisting of the next $\mu_{p+m-1}$ terms, etc. Finally, let $J_{p+1, p+1}$ be the 
set consisting of all the terms that remain after $J_{p+1, p+m}, J_{p+1, p+m-1}, \cdots$, $J_{p+1, p+2}$ have been defined.

1. Preliminaries. If $V$ is any set and $n$ is a positive integer, then we let ${ }^{n} V$ be the set of all $n$-termed sequences $x=\left\langle x_{0}, x_{1}, \cdots, x_{n-1}\right\rangle$ of elements of $V$. In particular, ${ }^{2} V$ if the universal equivalence relation over $V$. We let $\mathrm{id}_{V}$ be the identity relation over $V$, or the identity map of $V$ onto itself. If $V$ is a subset of the domain of the function $f$, then $f \uparrow V$ is the restriction of $f$ to $V$. If $\theta$ is an equivalence relation over $V$, then for $u \in V$ we let $u / \theta$ be the $\theta$-class to which $u$ belongs, and for $x \in{ }^{n} V$ we let $x / \theta=\left\langle x_{0} / \theta, x_{1}\left|\theta, \cdots, x_{n-1}\right| \theta\right\rangle$.

Since the indexing of the operations is usually not important, we often regard algebras simply as ordered pairs $A=\langle U, F\rangle$ consisting of a nonempty set $U$ and a set $F$ of operations over $U$, and we then write $\operatorname{Con}(U, F)$ for $\operatorname{Con}(A)$. If $X \subseteq{ }^{2} U$, then we let $\operatorname{con}_{A}(X)$ be the smallest congruence relation $\theta$ over $A$ with $X \leqq \theta$. If $X$ consists of a single ordered pair $\langle x, y\rangle$, then we write $\operatorname{con}_{A}(x, y)$ for $\operatorname{con}_{A}(X)$. Thus $\operatorname{con}_{A}(x, y)$ is the smallest congruence relation over $A$ that identifies $x$ and $y$. The following three statements hold for an arbitrary algebra $A=\left\langle U, f_{1}(i \in I)\right\rangle$.

(1.1) If the equivalence relation $\theta$ over $U$ identifies all the elements in the range of $f_{i}$ for each $i \in I$, then $\theta \in \operatorname{Con}(A)$.

(1.2) Suppose $x, y, z \in U$ and $z \neq x, y$, and let $\theta=\operatorname{con}_{A}(x, y)$. If $z$ does not belong to the range of any of the operations $f_{i}$, then $z / \theta=\{z\}$.

(1.3) Suppose $x, y \in U$, and let $\theta=\operatorname{con}_{A}(x, y)$. If neither $x$ nor $y$ belongs to the range of any of the operations $f_{i}$, then $x / \theta=\{x, y\}$.

The proof of (1.1) is trivial. Under the hypothesis of (1.2), $\phi=$ ${ }^{2}(U-\{z\}) \cup\{\langle z, z\rangle\}$ is a congruence relation over $A$ by (1.1), and therefore $\theta \subseteq \phi$, hence $z / \theta=\{z\}$. To prove (1.3) we use a similar argument, taking $\phi={ }^{2}(U-\{x, y\}) \cup{ }^{2}\{x, y\}$.

By a translation of an operation $p$ over $U$ we mean an operation $p^{\prime}$ obtained from $p$ by fixing some of the arguments. If $G$ is a set of operations over $U$, and if $G^{\prime}$ is the set of all unary translations of operations in $G$, then $\operatorname{Con}(U, G)=\operatorname{Con}\left(U, G^{\prime}\right)$. We let $P(A)$ be the set of all operations over $U$ that preserve all the congruence relations over $A$, and we let $P_{n}(A)$ be the set of all members of $P(A)$ that are of rank $n$. Obviously every algebraic function over $A$ belongs to $P(A)$, and every translation of a member of $P(A)$ belongs to $P(A)$. Our interest in these functions stems from the obvious fact that if $G$ is any set of operations over $U$ with $\operatorname{Con}(A)=\operatorname{Con}(U, G)$, then $G \subseteq P(A)$. 
We shall have occasion to work with partial algebras, where the maps are not necessarily defined for all values of the arguments (partial operations). The notion of a congruence relation over a partial algebra is assumed to be familiar, and the notation $\operatorname{con}_{A}(X)$ and $\operatorname{con}_{A}(x, y)$ will be applied also to these structures. Following [1], we call a congruence relation $\theta$ over $A$ strong if for every $i \in I$, and for every sequence $x$ in the domain of $f_{i}$, every sequence $y$ with $x / \theta=y / \theta$ also belongs to the domain of $f_{i}$. The notion of a free closure of a partial algebra $A^{\prime}=\left\langle U^{\prime}, f_{i}^{\prime}(i \in I)\right\rangle$ will play a crucial role. By this we mean an algebra $A=\left\langle U, f_{i}(i \in I)\right\rangle$ such that $A^{\prime}$ is a partial subalgebra of $A, A$ is generated by $U^{\prime}$, and every homomorphism of $A^{\prime}$ into another algebra $B$ can be extended to a homomorphism of $A$ into $B$. A more constructive description of free closures can be found e.g. in [1], §15 and in [3], §4.5. Following [3], call a partial algebra $B=\left\langle V, g_{i}(i \in I)\right\rangle$ a one-point extension of $A^{\prime}$ if there exist $y \in V-U^{\prime}$, $j \in I$, and $x \in{ }^{n} U^{\prime}$, where $n$ is the rank of $f_{j}$, such that

$$
V=U^{\prime} \cup\{y\}, \quad g_{j}=f_{j}^{\prime} \cup\{\langle x, y\rangle\},
$$

and $g_{i}=f_{i}^{\prime}$ whenever $j \neq i \in I$. We recall from [3], Corollary 4.5.6:

(1.4) $A$ is a free closure of $A^{\prime}$ iff for some ordinal $\alpha$ there exist partial algebras $B_{\xi}=\left\langle V_{\xi}, g_{\xi, i}(i \in I)\right\rangle(\xi \leqq \alpha)$ such that

(i) $B_{0}=A^{\prime}$ and $B_{\alpha}=A$.

(ii) For every ordinal $\xi<\alpha, B_{\xi+1}$ is a one-point extension of $B_{\xi}$.

(iii) For every limit ordinal $\lambda \leqq \alpha, B_{\lambda}$ is the union of the partial algebras $B_{\xi}$ with $\xi<\lambda$.

Suppose now that $A$ is a free closure of $A^{\prime}$. The following facts are well known, except possibly the last two.

(1.5) Suppose $i \in I$, and the rank of $f_{i}$ is $n$. If $x \in{ }^{n} U$ and $f_{i}(x) \in U^{\prime}$, then $x$ belongs to the domain of $f_{i}^{\prime}$.

(1.6) If $\phi \in \operatorname{Con}\left(A^{\prime}\right)$ and $\theta=\operatorname{con}_{A}(\phi)$, then $\theta$ is an extension of $\phi$; i.e., $\phi=\theta \cap{ }^{2} U^{\prime}$.

(1.7) For any strong congruence relation $\phi$ over $A^{\prime}, \phi \cup^{2}\left(U-U^{\prime}\right)$ is a congruence relation over $A$.

(1.8) If $\phi$ is a strong congruence relation over $A^{\prime}$, and if $\theta=$ $\operatorname{con}_{A}(\phi)$, then $x / \theta=x / \phi$ for all $x \in U^{\prime}$.

(1.9) For any elements $x, y \in U$ that are not both in $U^{\prime}, \operatorname{con}_{A}(x, y)$ identifies no two distinct members of $U^{\prime}$.

(1.10) If $A^{\prime}$ is not an algebra, then for each $x \in U$, the intersec- 
tion of all the relations $\operatorname{con}_{A}(x, y)$ with $y \in U-U^{\prime}$ is $\mathrm{id}_{U}$.

Proof of (1.5). Let $B_{\xi}(\xi \leqq \alpha)$ be as in (1.4). If $x$ is not in the domain of $f_{i}^{\prime}$, then there is an ordinal $\xi<\alpha$ such that $x$ is in the domain of $g_{\xi+1, i}$, but is not in the domain of $g_{\xi, i}$. It follows by the definition of one-point extensions that $f_{i}(x)=g_{\xi+1, i}(x)$ is the unique member of $V_{\xi+1}-V_{\xi}$, and therefore does not belong to $U^{\prime}$.

Proof of (1.6). The canonical homomorphism of $A^{\prime}$ onto $A^{\prime} / \phi$ can be extended to a homomorphism $h$ of $A$ onto the free closure of $A^{\prime} / \phi$ (or onto some other closure of $A^{\prime} / \phi$ ). The kernel $\psi$ of $h$ is a congruence relation over $A$ with $\phi \subseteq \theta \subseteq \psi$ and $\psi \cap{ }^{2} U^{\prime}=\phi$. Therefore, $\theta \cap{ }^{2} U^{\prime}=\phi$.

Proofs of (1.7) and (1.8). Let $\psi=\phi \cup^{2}\left(U-U^{\prime}\right)$. Consider any $i \in I$, let the rank of $f_{i}$ be $n$, and suppose $x, y \in{ }^{n} U$ and $x / \psi=y / \psi$. If either $x$ or $y$ belongs to the domain of $f_{i}^{\prime}$, then they both do, and $x / \phi=y / \phi$, hence the elements $f_{i}(x)=f_{i}^{\prime}(x)$ and $f_{i}(y)=f_{i}^{\prime}(y)$ are identified by $\phi$, and therefore also by $\psi$. If $x$ and $y$ are not in the domain of $f_{i}^{\prime}$, then $f_{i}(x)$ and $f_{i}(y)$ belong to $U-U^{\prime}$ by (1.5), and they are therefore identified by $\psi$. This proves (1.7), and (1.8) follows, since $\phi \subseteq \theta \leqq \psi$.

Proof of (1.9). Let $B_{\xi}(\xi \leqq \alpha)$ be as in (1.4), and observe that $A$ is a free closure of each of the partial algebras $B_{\xi}$. We may assume that $x$ and $y$ are distinct and do not both belong to $U^{\prime}$. Hence there exists $\xi<\alpha$ such that $V_{\xi+1}$ contains both $x$ and $y$, but $V_{\xi}$ contains only one of the two; say $x \in V_{\xi}, y \in V_{\xi+1}-V_{\xi}$. Then, since no sequence containing $y$ as one of its terms belongs to the domain of any of the operations $g_{\xi+1, i}$, we have

$$
\operatorname{con}_{B_{\xi+1}}(x, y)=\{\langle x, y\rangle,\langle y, x\rangle\} \cup \operatorname{id}_{V_{\xi+1}} \text {. }
$$

Now applying (1.6) with $A^{\prime}$ replaced by $B_{\xi+1}$ and with $\phi=\operatorname{con}_{B_{\xi+1}}(x, y)$, we get

$$
\begin{aligned}
{ }^{2} U^{\prime} \cap \operatorname{con}_{A}(x, y) & ={ }^{2} U^{\prime} \cap \operatorname{con}_{A}(\dot{\phi}) \\
& ={ }^{2} U^{\prime} \cap{ }^{2} V_{\xi+1} \cap \operatorname{con}_{A}(\phi) \\
& ={ }^{2} U^{\prime} \cap \phi=\mathrm{id}_{L^{\prime}} .
\end{aligned}
$$

Proof of (1.10). Again let $B_{\xi}(\xi \leqq \alpha)$ be as in (1.4). Observe that $\alpha$ must be a limit ordinal, for $B_{\xi+1}$ always lacks the closure property. In fact, if $y$ is the unique member of $V_{\xi+1}-V_{\xi}$, then no sequence that has $y$ as one of its terms belongs to the domain of any of the operations $g_{\xi+1, i}$. (Recall that we are excluding operations of rank 
zero. Statement (1.10) is false when there are no operations of positive rank.) Given $z, u \in U$ with $z \neq u$, we can therefore find $\xi<\alpha$ such that $x, z, u \in V_{\xi}$. Choosing $y \in U-V_{\xi}$, we apply (1.9) with $A^{\prime}$ replaced by $B_{\xi}$ to infer that $\operatorname{con}_{A}(x, y)$ does not identify $z$ and $u$.

2. A basic lemma. The result to be proved here is in fact the special case of our theorem in which the multiplicity type $\mu$ is of the form $\langle 0,0, \cdots, 0,1,0, \cdots\rangle$, with an additional condition imposed on the algebra.

Lemma. For any positive integer $k$ and any cardinal $m$, there exists an algebra $B=\langle V, g\rangle$ such that the rank of $g$ is $k$, there is no subset $G$ of $P_{k-1}(B)$ with $\operatorname{Con}(B)=\operatorname{Con}(V, G)$ and $|G|<m$, and there exist two distinct elements in $V$ that do not belong to the range of $g$.

We may assume that $k>1$, since the case $k=1$ is trivial. It is clear that if the conclusion of the lemma holds for a given value of $m$, then it holds for all smaller values, and iwe may therefore assume without loss of generality that $m$ is infinite.

We begin the proof of the lemma by recalling the basic construction from [5]. Choose a set $I$ with $|I|=m$; for convenience assume that $0 \notin I$, and let $I^{\prime}=I \cup\{0\}$. Choose distinct elements $a_{i}$, $b_{i}$ associated with the indices $i \in I^{\prime}$, and let $W$ be the set of all these elements $a_{i}$ and $b_{i}$. For $i \in I^{\prime}$ define $h_{i}\left(a_{0}\right)=a_{i}, h_{i}\left(b_{0}\right)=b_{i}$, and $h_{i}(x)=x$ whenever $x \in W-\left\{a_{0}, b_{0}\right\}$. As is shown in [5], the algebra $C=$ $\left\langle W, h_{i}(i \in I)\right\rangle$ has the property that there is no subset $G$ of $P(C)$, with $\operatorname{Con}(C)=\operatorname{Con}(W, G)$ and $|G|<m$. This, incidentally, proves the necessity of our inequality for $k=1$. However, we shall not make explicit use of this fact, but rather use some of the more technical properties of $C$ that are established in [5] in the process of proving the above assertion. These properties are stated in (2.1), (2.2), and (2.3) below.

(2.1) For all $x, y \in W-\left\{a_{0}, b_{0}\right\}$,

$$
\begin{aligned}
\operatorname{con}_{C}(x, y) & =\{\langle x, y\rangle,\langle y, x\rangle\} \cup \mathrm{id}_{W}, \\
\operatorname{con}_{C}\left(a_{0}, x\right) & ={ }^{2}\left(\{x\} \cup\left\{a_{i}: i \in I^{\prime}\right\}\right) \cup \mathrm{id}_{W}, \\
\operatorname{con}_{C}\left(b_{0}, x\right) & ={ }^{2}\left(\{x\} \cup\left\{b_{i}: i \in I^{\prime}\right\}\right) \cup \mathrm{id}_{W}, \\
\operatorname{con}_{C}\left(a_{0}, b_{0}\right) & =\bigcup_{i \in I^{\prime}}\left\{\left\langle a_{i}, b_{i}\right\rangle,\left\langle b_{i}, a_{i}\right\rangle\right\} \cup \operatorname{id}_{W} .
\end{aligned}
$$

(2.2) For any function $p$ in $P_{n}(C)$, either $p$ is constant, or else 
there exist $i \in I^{\prime}$ and $\kappa<n$ such that $p(x)=h_{i}\left(x_{\kappa}\right)$ for all $x \in{ }^{n} W$.

(2.3) For each $j \in I$ there exists an equivalence relation over $W$ that is not preserved by $h_{j}$, but is preseved by all the other operations $h_{i}$.

Statement (2.1) is the same as Lemma 2.2 of [5], and (2.2) combines Lemma 2.6 and Corollary 2.7 of that paper. To prove (2.3), consider the equivalence relation.

$$
{ }^{2}\left(W-\left\{a_{j}, b_{j}\right\}\right) \cup\left\{\left\langle a_{j}, a_{j}\right\rangle,\left\langle b_{j}, b_{j}\right\rangle\right\} .
$$

To construct the algebra $B$, choose a set $K$ that is disjoint from $W$ and has at least $m$ elements. With each index $i \in I$ associate a $(k-1)$-termed sequence $c^{i}$ of elements of $K$ in such a way that, for all $i, j \in I$ and $\kappa, \tau<k-1$,

$$
c_{\kappa}^{i}=c_{\tau}^{j} \text { iff } i=j \text { and } \kappa=\tau,
$$

and that there are infinitely many elements in $K$ that are not of the form $c_{n}^{i}$. Let $V^{\prime}=W \cup K$, and define the partial operation $g^{\prime}$ of rank $k$ over $V^{\prime}$ by letting

$$
g^{\prime}\left(c_{0}^{i}, c_{1}^{i}, \cdots, c_{k-2}^{i}, x\right)=h_{i}(x)
$$

for all $i \in I$ and $x \in W$, and letting $g^{\prime}$ be undefined in all other cases. Finally, let $B=\langle V, g\rangle$ be a free closure of the partial algebra $B^{\prime}=$ $\left\langle V^{\prime}, g^{\prime}\right\rangle$.

The proof of the lemma will be based on a rather detailed study of the functions in $P(B)$. Using Statements (2.4)-(2.11) below, we will show that if $G$ is a subset of $P_{k-1}(B)$ with $\operatorname{Con}(B)=\operatorname{Con}(V, G)$, then each of the functions $h_{i}(i \in I)$ is the restriction to $W$ of a unary translation of some member of $G$, and that only finitely many functions $h_{i}$ can be obtained in this manner from each member of $G$. Since the cardinal $m$ of $I$ is infinite, this will imply that $|G| \geqq m$.

(2.4) For every $p \in P_{n}(B)$, either $p\left({ }^{n} W\right) \subseteq W$ or $p\left({ }^{n} W\right) \cap W=\varnothing$.

Proof. Observe that the relation $\phi={ }^{2} W \cup \mathrm{id}{ }_{K}$ is a strong congruence relation over $B^{\prime}$, and therefore has an extension $\theta \in \operatorname{Con}(B)$ such that $x / \theta=x / \phi$ for all $x \in V^{\prime}$. Therefore, if $p(y) \in W$ for one sequence $y \in{ }^{n} W$, then for any other sequence $z \in{ }^{n} W$ we have $y / \theta=$ $z / \theta$, hence $p(y) \theta p(z)$, which implies that $p(z) \in W$.

(2.5) For every $p \in P_{1}(B)$, either $p\left(V-V^{\prime}\right) \subseteq V-V^{\prime}$ or $p$ is constant. 
Proof. Suppose there exists $x \in V-V^{\prime}$ with $p(x) \in V^{\prime}$. By (1.7), $\theta={ }^{2}\left(V-V^{\prime}\right) \cup \mathrm{id}_{V^{\prime}}$ is a congruence relation over $B$. Therefore, if $y \in V-V^{\prime}$, then $x \theta y$, hence $p(x) \theta p(y)$, which implies that $p(y)=p(x)$. Thus $p$ is constant on $V-V^{\prime}$. If $y \in V^{\prime}$, then $p(y)$ is in the relation $\operatorname{con}_{B}(y, z)$ to the element $p(z)=p(x)$ for every $z \in V-V^{\prime}$, and by (1.10) this implies that $p(y)=p(x)$.

It is an immediate consequence of (2.5) that if $p \in P_{n}(B), x \in{ }^{n} V$, and $p(x) \in V^{\prime}$, then those terms of $x$ that do not belong to $V^{\prime}$ can be replaced by arbitrary members of $V$,-in particular by members of $W$, - without changing the value of the function. This fact will be used several times.

(2.6) For every $p \in P_{1}(B)$, either $p(V) \cap K=\varnothing$ or $p=\mathrm{id}_{V}$ or $p$ is constant.

Proof. Suppose there exists $x \in V$ with $p(x) \in K$. Since the element $d=p(x)$ belongs to $V^{\prime}$ and is not in the range of $g^{\prime}$, it follows from (1.5) that $d$ is not in the range of $g$. First suppose $d \neq x$. For any $y \in V-\{d\}$, if we let $\theta=\operatorname{con}_{B}(x, y)$, then it follows by (1.2) that $d / \theta=\{d\}$. Since $p(y) \theta d$, this implies that $p(y)=d$. To show that $p(d)$ is also equal to $d$, we merely observe that, for each $y \in V-$ $\{d\}, \operatorname{con}_{B}(y, d)$ identifies $p(d)$ and $d$, and then apply (1.10).

Now suppose $d=x$. By (1.3), if $e \in K-\{d\}$, then the $\operatorname{con}_{B}(d, e)$ class of $d$ consists of $d$ and $e$ alone, whence it follows that $p(e)=d$ or $p(e)=e$. If $p(e)=d$ for one such element $e$, then $p$ is constant by the first part of the proof. We may therefore assume that $p$ maps every member of $K$ onto itself. To show that in this case $p(y)=y$ for all $y \in V$, it is sufficient to prove that the intersection of all the relations $\operatorname{con}_{B}(y, e)$ with $e \in K$ is $\operatorname{id}_{V}$.

It is thus sufficient to show that for any two distinct elements $z, u \in V$, there exists $e \in K$ such that $\operatorname{con}_{B}(y, e)$ does not identify $z$ and $u$. Let $L$ be the set consisting of all the elements $c_{\kappa}^{i}$ with $i \in I$ and $\kappa<k-1$. There exists a finite subset $M$ of $K-L$ such that $y, z$, and $u$ are in the subalgebra of $B$ generated by $W \cup L \cup M$. Let $\rho^{\prime}$ be the map of $V^{\prime}$ into $V^{\prime}$ that takes each member of $W \cup L \cup M$ into itself, and all the elements of $K-(L \cup M)$ into $y$. This map $\rho^{\prime}$ is an endomorphism of the partial algebra $B^{\prime}$, for every sequence in the domain of $g^{\prime}$ is mapped onto itself by $\rho^{\prime}$, and every element in the range of $g^{\prime}$ is also mapped onto itself. Therefore, $\rho^{\prime}$ extends to an endomorphism $\rho$ of $B$. Every element in the subalgebra of $B$ generated by $W \cup L \cup M$ is mapped onto itself by $\rho$, and in particular, this is true of the elements $y, z$, and $u$. We now make use of the fact that $K-L$ is infinite to choose an element $e$ in $K$ that is not in $L \cup M$. Since $\rho(e)=y=\rho(y), \operatorname{con}_{B}(y, e)$ is contained in the kernel 
of $\rho$, and since $\rho(z) \neq \rho(u)$, this shows that $\operatorname{con}_{B}(y, e)$ does not identify $z$ and $u$. $i \in I^{\prime}$.

(2.7) If $x \in V-V^{\prime}$ and $\theta=\operatorname{con}_{B}\left(b_{0}, x\right)$, then $a_{i} / \theta=\left\{a_{i}\right\}$ for all

Proof. We first note that if $p$ is any algebraic function of rank one over $B$, and if $p\left(b_{0}\right)=a_{i}$ for some $i \in I^{\prime}$, then $p$ is constant. In fact, if there is a nonconstant function $p$ with this property, then there is a simplest such function. Since $p$ is neither constant nor the identity function, there exist algebraic functions $q_{0}, q_{1}, \cdots, q_{k-1}$ of rank one over $B$, all of them simpler than $p$, such that

$$
p(y)=g\left(q_{0}(y), q_{1}(y), \cdots, q_{k-1}(y)\right)
$$

for all $y \in V$. In particular,

$$
a_{i}=g\left(q_{0}\left(b_{0}\right), q_{1}\left(b_{0}\right), \cdots, q_{k-1}\left(b_{0}\right)\right) .
$$

By (1.5), $g$ can be replaced by $g^{\prime}$ in this formula, and we infer from the definition of $g^{\prime}$ that $q_{\kappa}\left(b_{0}\right)=c_{\kappa}^{i}$ for $\kappa<k-1$, and that $q_{k-1}\left(b_{0}\right)$ is either $a_{i}$ or $a_{0}$. We conclude that $q_{k-1}$ must be constant, and by (2.6) the other functions $q_{\kappa}$ are also constant. Thus $p$ must be constant, contrary to our assumption.

Now suppose $a_{i} \theta y$. Then there exist elements $z_{0}, z_{1}, \cdots, z_{n} \in V$, and algebraic functions $p_{0}, p_{1}, \cdots, p_{n-1}$ of one variable over $B$ such that $z_{0}=a_{i}, z_{n}=y$, and $\left\{z_{\kappa}, z_{\kappa+1}\right\}=\left\{p_{k}\left(b_{0}\right), p_{\kappa}(x)\right\}$ for $\kappa=0,1, \cdots, n-1$. We have shown that if $p_{0}\left(b_{0}\right)=a_{i}$, then $p_{0}$ is constant, and by (2.6), $p_{0}(x)=a_{i}$ also implies that $p_{0}$ is constant. Thus, in either case, $a_{i}=$ $z_{0}=z_{1}$. By repeating this argument we infer that $z_{\kappa}=a_{i}$ for all $\kappa \leqq$ $n$ and, therefore, $y=a_{i}$.

(2.8) If $p \in P_{n}(B)$, and if $p\left({ }^{n} W\right) \subseteq W$ and $p$ is constant on $W$, then $p$ is constant.

Proof. By symmetry we may assume that $p(x)=a_{i}$ for all $x \in{ }^{n} W$. In particular then, $p\left(b_{0}, b_{0}, \cdots, b_{0}\right)=a_{i}$. Choosing $y \in V-V^{\prime}$, we infer that $\operatorname{con}_{B}\left(b_{0}, y\right)$ identifies $p(y, y, \cdots, y)$ and $a_{i}$, and by (2.7) this implies that $p(y, y, \cdots, y)=a_{i}$. From this the conclusion follows by (2.5).

(2.9) Suppose $p \in P_{n}(B)$ and $x \in{ }^{n-1} V$, and for $z \in V$ let

$$
p^{\prime}(z)=p\left(x_{0}, x_{1}, \cdots, x_{n-2}, z\right) .
$$

If $p^{\prime} \uparrow W=h_{i}$ for some $i \in I^{\prime}$, then there exists $y \in{ }^{n-1} K$ such that, for all $z \in W$, 


$$
p^{\prime}(z)=p\left(y_{0}, y_{1}, \cdots, y_{n-2}, z\right) .
$$

Proof. By (2.5), any term $x_{\kappa}$ that belongs to $V-V^{\prime}$ can be replaced by an arbitrary member of $V$ without changing the function $p^{\prime} \uparrow W$. It is therefore sufficient to show that if a term $x_{\kappa}$ that belongs to $W$ is replaced by some other member of $V$, then the values of $p^{\prime}$ on $W$ do not change. It is clearly sufficient to consider the case when $n=2$ and $x$ is therefore simply an element in $W$. We may further assume that $x \neq a_{0}, a_{i}$, for if $x=a_{0}$ or $x=a_{i}$, then the $a$ 's and the $b$ 's can be interchanged in the argument that follows.

For $y \in V$ let $q(y)=p\left(y, a_{0}\right)$. Then $q(x)=a_{i} \in W$, whence it follows that $q$ maps $W$ into itself. Thus $q \uparrow W$ is a member of the set $P_{1}(C)$, and must therefore be constant by (2.2), since it cannot be one of the functions $h_{j}$. By (2.8), $q$ is therefore constant, i.e., we have $p\left(y, a_{0}\right)=a_{i}$ for all $y \in V$.

For all $y, z \in V$ let $p_{y}(z)=p(y, z)$. Then $p_{y}\left(a_{0}\right)=a_{i}$, and therefore $p_{y}$ maps $W$ into itself. Using (2.2) we infer that each of the functions $p_{y} \uparrow W$ is either equal to $h_{i}$, or else is constant. If $p_{y}$ is constant, then $p\left(y, b_{0}\right)=a_{i}$, while $p\left(x, b_{0}\right)=b_{i}$. For $y \in V-V^{\prime}$ this cannot be the case, for by (1.9) $\operatorname{con}_{B}(x, y)$ does not identify $a_{i}$ and $b_{i}$. For such values of $y$ we must therefore have $p(y, z)=h_{i}(z)$ for all $z \in W$. But by (2.5), if this holds for one value of $y$ in $V-V^{\prime}$, then it holds for all $y \in V$.

(2.10) If $n<k$ and $p \in P_{n}(B)$, then there are only finitely many indices $i \in I$ such that some unary translation of $p$ agrees with $h_{i}$ on $W$.

Proof. Suppose $x, y \in{ }^{n-1} V$, and for $z \in V$ let

$$
\begin{aligned}
p^{\prime}(z) & =p\left(x_{0}, x_{1}, \cdots, x_{n-2}, z\right), \\
p^{\prime \prime}(z) & =p\left(y_{0}, y_{1}, \cdots, y_{n-2}, z\right) .
\end{aligned}
$$

Suppose each of the functions $p^{\prime}$ and $p^{\prime \prime}$ agrees on $W$ with some function $h_{i}$. By (2.9) we may assume that $x, y \in{ }^{n-1} K$. Let

$$
X=\left\{\left\langle x_{k}, y_{k}\right\rangle: \kappa=0,1, \cdots, n-2\right\},
$$

and let $\theta=\operatorname{con}_{B}(X)$. It will be shown that $\theta$ identifies no two distinct members of $W$. Since $p^{\prime}(z) \theta p^{\prime \prime}(z)$ for all $z \in V$, this implies that $p^{\prime}$ and $p^{\prime \prime}$ agree on $W$. Thus by fixing all but the last coordinate, and restricting the resulting function to $W$, we obtain at most one function $h_{i}$. The same is of course true of the other coordinates, and the total number of functions $h_{i}$ obtainable in this manner is therefore finite, - in fact at most $n$.

Let $\theta^{\prime}$ be the smallest equivalence relation over $V^{\prime}$ with $X \cong \theta^{\prime}$. 
Then $z / \theta^{\prime}=\{z\}$ for all $z \in W$, and $\theta=\operatorname{con}_{B}\left(\theta^{\prime}\right)$. Observe that no two distinct sequences in the domain of $g^{\prime}$ are identified by $\theta^{\prime}$. In fact, such sequences have the form

$$
\left\langle c_{0}^{i}, c_{1}^{i}, \cdots, c_{k-2}^{i}, x\right\rangle, \quad\left\langle c_{0}^{j}, c_{1}^{j}, \cdots, c_{k-2}^{j}, y\right\rangle
$$

with $i, j \in I$ and $x, y \in W$. If $x \neq y$, then $x$ and $y$ are not identified by $\theta^{\prime}$, and if $i \neq j$, then the $2 k-2$ distinct elements $c_{\kappa}^{i}$ and $c_{\kappa}^{j}$ cannot all occur as coordinates of members of $X$, and therefore there is some $\kappa<k-1$ such that $\theta^{\prime}$ does not identify $c_{k}^{i}$ and $c_{k}^{j}$. We infer that $\theta^{\prime}$ is a congruence relation over $B^{\prime}$, so that by (1.6) $\theta$ is an extension of $\theta^{\prime}$. Since $\theta^{\prime}$ identifies no two distinct members of $W$, it follows that the same is true of $\theta$, as was to be shown.

(2.11) If $p \in P_{1}(B)$, and if there exists an equivalence relation $\phi$ over $W$ such that the equivalence relation $\dot{\phi} \cup^{2}\left(V-V^{\prime}\right) \cup$ id $d_{K}$ is not preserved by $p$, then $p \uparrow W=h_{i}$ for some $i \in I$.

Proof. Let $\theta=\dot{\phi} \cup^{2}\left(V-V^{\prime}\right) \cup \mathrm{id}_{K}$. By (2.4) and (2.6), either $p(W) \subseteq W$ or $p(W) \subseteq V-V^{\prime}$. In the latter case, however, $p$ would preserve $\theta$, because by (2.5), $p$ also maps $V-V^{\prime}$ into $V-V^{\prime}$. Thus we must have $p(W) \subseteq W$. Since every congruence relation over $C$ extends to a congruence relation over $B$, it follows that $p \nmid W \in P_{1}(C)$. Consequently, by (2.2), either $p \uparrow W$ is constant, or else it is equal to one of the functions $h_{i}$. However, if $p \uparrow W$ were constant, then by (2.8), $p$ would be constant, and would therefore preserve $\theta$. Thus $p \nmid W=h_{i}$ for some $i \in I^{\prime}$. The case $i=0$ is easily ruled out, for if $p(x)=x$ for all $x \in W$, then $p$ preserves $\theta$. Thus $i \in I$.

To complete the proof of the lemma, suppose $G$ is a subset of $P_{k-1}(B)$ with $\operatorname{Con}(B)=\operatorname{Con}(V, G)$, and let $G^{\prime}$ be the set of all unary translations of members of $G$. For each $j \in I$ there exists by (2.3) an equivalence relation $\phi$ over $W$ that is not preserved by $h_{j}$, but is preserved by all the other operations $h_{i}$. Since $\phi$ is not a congruence relation over $C$, it does not extend to a congruence relation over $B$ and, in particular, the relation $\theta=\dot{\phi} \cup^{2}\left(V-V^{\prime}\right) \cup$ id $_{K}$ is not a congruence relation over $B$. Therefore, there must be an operation $p$ in $G^{\prime}$ that does not preserve $\theta$, and by (2.11) we have $p \nmid W=h_{i}$ for some $i \in I$. We claim that, in fact, $i=j$. To see that, consider two elements $x, y \in V$ such that $x \theta y$ but not $p(x) \theta p(y)$. Clearly $x$ and $y$ are either both in $W$ or both in $V-V^{\prime}$. In the latter case, however, $p(x)$ and $p(y)$ would also belong to $V-V^{\prime}$ according to (2.5), and would therefore be identified by $\theta$. Thus, $x, y \in W$ and $x \phi y$, but not $h_{i}(x) \phi h_{i}(y)$. In other words, $h_{i}$ does not preserve $\phi$, and therefore $i=j$. 
We have shown that for each $j \in I$ there exists $p_{j} \in G^{\prime}$ such that $p_{j} \uparrow W=h_{j}$. By (2.10), only finitely many of these functions $p_{j}$ can be translations of the same function in $G$. Since the cardinal $m$ of $I$ is infinite, it follows that $|G| \geqq m$.

The final condition in the lemma is obviously satisfied. In fact, $a_{0}$ and $b_{0}$ do not belong to the range of $g$, and neither do the members of $K$.

3. The proof of the theorem. Suppose $\mu$ and $\mu^{\prime}$ are two multiplicity types, $k$ is a positive integer, and

$$
\sum_{n=k}^{\infty} \mu_{n}>\sum_{n=k}^{\infty} \mu_{n}^{\prime}
$$

Let

$$
N=\sum_{n=k}^{\infty} \mu_{n},
$$

and choose an infinite cardinal $m$ such that

$$
m>\sum_{n=1}^{\infty} \mu_{n}^{\prime}
$$

Choose a set $I$ of cardinality $N$, and for each $i \in I$ an algebra $B_{i}=$ $\left\langle V_{i}, g_{i}\right\rangle$ that satisfies all the conditions of the lemma, i.e., the rank of $g_{i}$ is $k$, there is no set $G \subseteq P_{k-1}\left(B_{i}\right)$ such that $\operatorname{Con}\left(B_{i}\right)=\operatorname{Con}\left(V_{i}, G\right)$ and $|G|<m$, and there are two distinct elements in $V_{i}$ that do not belong to the range of $g_{i}$. Assuming that the sets $V_{i}$ are pairwise disjoint, let $V^{\prime}$ be their union, and consider the partial algebra

$$
B^{\prime}=\left\langle V^{\prime}, g_{i}(i \in I)\right\rangle \text {. }
$$

Finally, let $A=\left\langle U, f_{i}(i \in I)\right\rangle$ be a free closure of $B^{\prime}$. Thus $A$ is an algebra with $N k$-ary operations, and there clearly exists an algebra of the multiplicity type $\mu$ whose congruence lattice is equal to Con $(A)$. To complete the proof it is therefore sufficient to show that there is no algebra of the multiplicity type $\mu^{\prime}$ whose congruence lattice is equal to $\operatorname{Con}(A)$. We shall in fact prove the stronger statement that if $G$ is any subset of $P(A)$ with $\operatorname{Con}(A)=\operatorname{Con}(U, G)$, then either $|G| \geqq m$, or else $G$ has at least $N$ members of rank $k$ or more. The information about the functions in $P(A)$ that is needed for this purpose is contained in (3.1)-(3.4) below.

(3.1) If $p \in P_{n}(A)$ and $i \in I$, then either $p\left({ }^{n} V_{i}\right) \subseteq V_{i}$ or $p\left({ }^{n} V_{i}\right) \cap$ $V_{i}=\varnothing$.

Proof. Observe that $\theta={ }^{2} V_{i} \cup{ }^{2}\left(U-V_{i}\right)$ is a congruence relation 
over $A$. In fact, for $j \neq i$ the range of $f_{j}$ is contained in $U-V_{i}$ by (1.5). If the sequences $x, y \in{ }^{k} U$ are identified by $\theta$, then either $x, y \in{ }^{k} V_{i}$, in which case $f_{i}(x)$ and $f_{i}(y)$ belong to $V_{i}$, or else $x_{\kappa}, y_{\kappa} \in U-$ $V_{i}$ for some $\kappa<k$, and therefore $f_{i}(x)$ and $f_{i}(y)$ are both in $U-V^{\prime}$ by (1.5). In either case, $f_{i}(x) \theta f_{i}(y)$.

Now suppose $p(x) \in V_{i}$ for some $x \in{ }^{n} V_{i}$. For every $y \in{ }^{n} V_{i}$ we then have $p(x) \theta p(y)$, hence $p(y) \in V_{i}$. Thus $p\left({ }^{n} V_{i}\right) \subseteq V_{i}$.

(3.2) If $p \in P_{1}(A)$, and if there exist $i \in I$ and $x \in U-V_{i}$ such that $p(x) \in V_{i}$, then $p$ is constant.

Proof. It is easy to check that $\theta={ }^{2}\left(U-V_{i}\right) \cup i d_{V_{i}}$ is a congruence relation over $A$. Therefore, if $y \in U-V_{i}$, then $p(x) \theta p(y)$, hence $p(y)=p(x)$. Thus $p$ is constant on $U-V_{i}$, say $p(y)=d$ for all $y \in U-V_{i}$. To show that this equation also holds for $y \in V_{i}$, observe that, for each $z \in U-V_{i}, p(y)$ is in the relation $\operatorname{Con}_{A}(y, z)$ to the element $p(z)=d$, and that by (1.10) the intersection of these relations is $\mathrm{id}_{U}$.

The same observation applies here as was made concerning (2.5): If $p \in P_{n}(A), x \in{ }^{n} U$, and $p(x) \in V_{i}$, then those terms of $x$ that do not belong to $V_{i}$ can be replaced by arbitrary members of $U$ without changing the value of the function.

(3.3) If $p \in P_{n}(A), i, j \in I, i \neq j, p\left({ }^{n} V_{i}\right) \subseteq V_{i}$, and $p\left({ }^{n} V_{j}\right) \subseteq V_{j}$, then there exists $\kappa<n$ such that $p(x)=x_{\kappa}$ for all $x \in{ }^{n} V_{i} \cup{ }^{n} V_{j}$.

Proof. First suppose $n=1$. If $x \in V_{i}$ and $y \in V_{j}$, then $p(x)$ and $p(y)$ are identified by $\operatorname{con}_{A}(x, y)$, and by $(1.6)$ they are therefore also identified by $\operatorname{con}_{B^{\prime}}(x, y)$. It is easy to check that

$$
\operatorname{con}_{B^{\prime}}(x, y)=\{\langle x, y\rangle,\langle y, x\rangle\} \cup \operatorname{id}_{V^{\prime}} .
$$

Therefore, $p(x)=x$ and $p(y)=y$.

Now suppose $n>1$, and assume that the statement holds whenever $n$ is replaced by a smaller integer. Choose elements $x \in V_{i}$ and $y \in V_{j}$ that do not belong to the ranges of $g_{i}$ and $g_{j}$, and therefore do not belong to the range of any of the operations of $A$. The elements $p(x, x, \cdots, x)$ and $p(y, y, \cdots, y)$ belong to $V_{i}$ and $V_{j}$, respectively, and are identified by $\operatorname{con}_{A}(x, y)$, whence it follows that $p(x, x, \cdots, x)=x$ and $p(y, y, \cdots, y)=y$. Therefore, the element $p(x, x, \cdots, x, y)$ is identified with $x$ by $\operatorname{con}_{A}(x, y)$, and so by (1.3) is equal to either $x$ or $y$.

Case 1. $p(x, x, \cdots, x, y)=y$. By (3.2) this implies that $p\left(z_{0}\right.$, $\left.z_{1}, \cdots, z_{n-2}, y\right)=y$ for all $z \in{ }^{n-1} U$. Therefore, $p\left(z_{0}, z_{1}, \cdots, z_{n-2}, x\right)$ is 
identified with $y$ by $\operatorname{con}_{A}(x, y)$, and is therefore equal to either $x$ or $y$.

Suppose, for some $z \in^{n-1} U, p\left(z_{0}, z_{1}, \cdots, z_{n-2}, x\right)=y$. Then, by (3.2), $p\left(z_{0}, z_{1}, \cdots, z_{n-2}, u\right)=y$ for all $u \in U$. If $z_{k} \neq y$, then by (1.2), $\operatorname{con}_{A}\left(x, z_{\kappa}\right)$ does not identify $y$ with any other element of $U$, and we can therefore replace $z_{\kappa}$ by $x$ without changing the value of the function. Thus if we let $z_{\kappa}^{\prime}=x$ when $z_{\kappa} \neq y$ and $z_{\kappa}^{\prime}=y$ if $z_{\kappa}=y$, then $p\left(z_{0}^{\prime}, z_{1}^{\prime}, \cdots, z_{n-2}^{\prime}, u\right)=y$. Consequently $p(x, x, \cdots, x, u)$ is identified with $y$ by $\operatorname{con}_{A}(x, y)$, and must therefore be equal to either $x$ or $y$. From the fact that $p(x, x, \cdots, x, y)=y$, we infer that for $u \neq x p(x, x, \cdots$, $x, u$ ) cannot be equal to $x$, because by $(1.2) \operatorname{con}_{A}(y, u)$ does not identify $x$ and $y$. Therefore, $p(x, x, \cdots, x, u)=y$ whenever $u \neq x$, while $p(x, x, \cdots, x)=x$. However, this is impossible because, by (1.10), the intersection of all the congruence relations $\operatorname{con}_{A}(x, u)$ with $u \neq x$ is equal to $i d_{U}$, and therefore does not identify $x$ and $y$. We have therefore shown that, for all $z \in{ }^{n-1} U, p\left(z_{0}, z_{1}, \cdots, z_{n-2}, x\right)=x$. From this and the fact that $p\left(z_{0}, z_{1}, \cdots, z_{n-2}, y\right)=y$ we infer by (3.1) that the map $u \rightarrow p\left(z_{0}, z_{1}, \cdots, z_{n-2}, u\right)$ takes $V_{i}$ into $V_{i}$ and $V_{j}$ into $V_{j}$, and by the case $n=1$ it follows that $p\left(z_{0}, z_{1}, \cdots, z_{n-2}, u\right)=u$ for all $u \in$ $V_{i} \cup V_{j}$. In particular, therefore, $p(z)=z_{n-1}$ for all $z \in{ }^{n} V_{i} \cup{ }^{n} V_{j}$.

Case 2. $p(x, x, \cdots, x, y)=x$. By (3.1), the map $z \rightarrow p\left(z_{0}, z_{1}, \cdots\right.$, $\left.z_{n-2}, y\right)$ takes ${ }^{n-1} V_{i}$ into $V_{i}$, and since $p(y, y, \cdots, y)=y$, this map also takes ${ }^{n-1} V_{j}$ into $V_{j}$. By the inductive hypothesis it follows that there exists $\kappa<n-1$ such that $p\left(z_{0}, z_{1}, \cdots, z_{n-2}, y\right)=z_{\kappa}$ for all $z \epsilon$ ${ }^{n-1} V_{i} \cup{ }^{n-1} V_{j}$. For a fixed sequence $z \in{ }^{n} V_{i}$, the map $u \rightarrow p\left(z_{0}, z_{1}, \cdots\right.$, $z_{n-2}, u$ ) is constant by (3.2), because it takes $y$ into the element $z_{\kappa}$ of $V_{i}$. Therefore,

$$
p(z)=p\left(z_{0}, z_{1}, \cdots, z_{n-2}, y\right)=z_{k} .
$$

For a sequence $z \in{ }^{n} V_{j}$, the elements $p\left(z_{0}, z_{1}, \cdots, z_{n-2}, x\right)$ and $p\left(z_{0}\right.$, $\left.z_{1}, \cdots, z_{n-2}, y\right)=z_{\kappa}$ are identified by $\operatorname{con}_{A}(x, y)$. In the particular case when $z_{\kappa}$ is an element of $V_{j}$ that is different from $y$ and is not in the range of any one of the basic operations of $A$ it follows by (1.2) that $p\left(z_{0}, z_{1}, \cdots, z_{n-2}, x\right)=z_{k}$. Therefore, by (3.1), $p\left(z_{0}, z_{1}, \cdots, z_{n-2}, x\right) \in$ $V_{j}$ for every $z \in{ }^{n} V_{j}$, and by (3.2) we can therefore replace $x$ by any member of $U$ without changing the value of the function. In particular, therefore,

$$
p(z)=p\left(z_{0}, z_{1}, \cdots, z_{n-2}, y\right)=z_{\kappa} .
$$

(3.4) Suppose $p \in P_{n}(A), i \in I$, and $\phi$ is an equivalence relation over $V_{i}$. If $\phi \cup{ }^{2}\left(U-V_{i}\right)$ is not preserved by $p$, then $p\left({ }^{n} V_{i}\right) \subseteq V_{i}$, and $p \uparrow^{n} V_{i}$ does not preserve $\phi$.

Proof. Let $\theta=\phi \cup{ }^{2}\left(U-V_{i}\right)$. If $p$ does not map ${ }^{n} V_{i}$ into $V_{i}$, 
then by (3.1), $p\left({ }^{n} V_{i}\right) \subseteq U-V_{i}$. It follows that the range of $p$ is contained in $U-V_{i}$, for if there were a sequence $x$ in ${ }^{n} U$ with $p(x) \in V_{i}$, then by (3.2) there would be such a sequence in ${ }^{n} V_{i}$. But this means that the whole range of $p$ is contained in one $\theta$-class, and therefore $p$ preserves $\theta$. Thus $p\left({ }^{n} V_{i}\right) \subseteq V_{i}$.

Now suppose $\phi$ is preserved by $p \uparrow^{n} V_{i}$. Considering any two sequences $x, y \in{ }^{n} U$ with $x / \theta=y / \theta$, we wish to show that $p(x) \theta p(y)$. This is certainly true if both $p(x)$ and $p(y)$ are in $U-V_{i}$, and we may therefore assume that $p(x) \in V_{i}$. Observe that, for each $\kappa<$ $n, x_{\kappa}$ and $y_{\kappa}$ are either both in $V_{i}$ or both in $U-V_{i}$; for notational convenience assume that $x_{\kappa}, y_{\kappa} \in V_{i}$ for $\kappa<\tau$ and $x_{\kappa}, y_{\kappa} \in U-V_{i}$ for $\tau \leqq \kappa<n$. For $z \epsilon^{*} U$ let

$$
p^{\prime}(z)=p\left(z_{0}, z_{1}, \cdots, z_{\tau-1}, y_{*}, \cdots, y_{n-1}\right),
$$

and observe that $p^{\prime}\left(x_{0}, x_{1}, \cdots, x_{\varepsilon-1}\right)=p(x) \in V_{i}$ by $(3.2)$, hence $p^{\prime}\left({ }^{\tau} V_{i}\right) \subseteq$ $V_{i}$ by (3.1). In particular,

$$
p(y)=p^{\prime}\left(y_{0}, y_{1}, \cdots, y_{--1}\right) \in V_{i} .
$$

Let $x_{\kappa}^{\prime}=x_{\kappa}$ and $y_{\kappa}^{\prime}=y_{\kappa}$ for $\kappa<\tau$, and choose $x_{\kappa}^{\prime}=y_{\kappa}^{\prime} \in V_{i}$ for $\tau \leqq \kappa<$ $n$. Then $x^{\prime} / \theta=y^{\prime} / \theta$ and, by (3.2), $p\left(x^{\prime}\right)=p(x)$ and $p\left(y^{\prime}\right)=p(y)$. Consequently $p(x) \phi p(y)$, and therefore $p(x) \theta p(y)$. Thus $p$ preserves $\theta$. This contradiction completes the proof of (3.4).

To complete the proof of the theorem, suppose $G \subseteq P(A),|G|<$ $m$, and $\operatorname{Con}(A)=\operatorname{Con}(U, G)$. For each $i \in I$ let $G_{i}$ be the set of all functions $p$ in $G$ such that, for some equivalence relation $\phi$ over $V_{i}$, $p$ does not preserve $\phi \cup \cup^{2}\left(U-V_{i}\right)$. Also let $G_{i}^{\prime}$ be the set of all restrictions of functions in $G_{i}$ to $V_{i}$. By (3.4), the functions in $G_{i}^{\prime}$ are operations over $V_{i}$, and $\operatorname{Con}\left(B_{i}\right)=\operatorname{Con}\left(V_{i}, G_{i}^{\prime}\right)$. In fact, every congruence relation $\phi$ over $B_{i}$ extends to a congruence relation $\theta$ over $A$, and since the members of $G_{i}$ preserve $\theta$, their restriction to $V_{i}$ obviously preserve $\phi$. On the other hand, if the equivalence relation $\phi$ over $V_{i}$ is not a congruence relation over $B_{i}$, then the relation $\theta=\phi \cup^{2}\left(U-V_{i}\right)$ is not a congruence relation over $A$. Hence there is some member $p$ of $G_{i}$ that does not preserve $\theta$, and by (3.4) this implies that the restriction of $p$ to $V_{i}$ does not preserve $\phi$. Since the algebras $B_{i}$ satisfy the conditions of the lemma, there exists for each $i \in I$ an operation in $G_{i}$ whose rank is at least $k$. Finally, by (3.3) and (3.4), the sets $G_{i}$ must be pairwise disjoint, and we conclude that there must be at least $N$ operations in $G$ whose rank is $k$ or more. This completes the proof.

\section{REFERENCES}

1. G. A. Grätzer, Universal Algebra, Van Nostrand, 1968. 
2. B. Jónsson, Multiplicity types of algebras and their congruence lattices, Notices Amer. Math. Soc., 19 (1972), A-569.

3. - Topics in Universal Algebra, Lecture Notes in Mathematics, Vol. 250, Springer-Verlag, 1972.

4. T. P. Whaley, Comparison of finite types by congruence relations, Notices Amer. Math. Soc., 18 (1971), A-225.

5. - Multiplicity types and congruence relations in universal algebra, Pacific J. Math., 39 (1971), 261-268.

Received October 6, 1972. The work of the first author was supported in part by National Science Foundation grants GP-29129 and GP-29129Al, and the work of the second author by a Research Corporation grant.

VANDERBILT UNIVERSITY

AND

Southwestern at Memphis 


\section{PACIFIC JOURNAL OF MATHEMATICS}

\section{EDITORS}

RICHARD ARENS (Managing Editor)

University of California

Los Angeles, California 90024

\section{R. A. Beaumont \\ University of Washington \\ Seattle, Washington 98105}

\section{J. DugundjI*}

Department of Mathematics University of Southern California Los Angeles, California 90007

D. Gilbarg and J. Milgram

Stanford University

Stanford, California 94305

\section{ASSOCIATE EDITORS}
E. F. BeCKenBaCH
B. H. NeumanN
F. WOLF
K. YoSHIDA

\section{SUPPORTING INSTITUTIONS}

\author{
UNIVERSITY OF BRITISH COLUMBIA \\ CALIFORNIA INSTITUTE OF TECHNOLOGY \\ UNIVERSITY OF CALIFORNIA \\ MONTANA STATE UNIVERSITY \\ UNIVERSITY OF NEVADA \\ NEW MEXICO STATE UNIVERSITY \\ OREGON STATE UNIVERSITY \\ UNIVERSITY OF OREGON \\ OSAKA UNIVERSITY
}

\author{
UNIVERSITY OF SOUTHERN CALIFORNIA \\ STANFORD UNIVERSITY \\ UNIVERSITY OF TOKYO \\ UNIVERSITY OF UTAH \\ WASHINGTON STATE UNIVERSITY \\ UNIVERSITY OF WASHINGTON

$* * *$
$*$
AMERICAN MATHEMATICAL SOCIETY
NAVAL WEAPONS CENTER

The Supporting Institutions listed above contribute to the cost of publication of this Journal, but they are not owners or publishers and have no responsibility for its content or policies.

Mathematical papers intended for publication in the Pacific Journal of Mathematics should be in typed form or offset-reproduced, (not dittoed), double spaced with large margins. Underline Greek letters in red, German in green, and script in blue. The first paragraph or two must be capable of being used separately as a synopsis of the entire paper. Items of the bibliography should not be cited there unless absolutely necessary, in which case they must be identified by author and Journal, rather than by item number. Manuscripts, in duplicate if possible, may be sent to any one of the four editors. Please classify according to the scheme of Math. Rev. Index to Vol. 39. All other communications to the editors should be addressed to the managing editor, or Elaine Barth, University of California, Los Angeles, California, 90024.

100 reprints are provided free for each article, only if page charges have been substantially paid. Additional copies may be obtained at cost in multiples of 50 .

The Pacific Journal of Mathematics is issued monthly as of January 1966. Regular subscription rate: $\$ 60.00$ a year (6 Vols., 12 issues). Special rate: $\$ 30.00$ a year to individual members of supporting institutions.

Subscriptions, orders for back numbers, and changes of address should be sent to Pacific Journal of Mathematics, 103 Highland Boulevard, Berkeley, California, 94708.

PUBLISHED BY PACIFIC JOURNAL OF MATHEMATICS, A NON-PROFIT CORPORATION

Printed at Kokusai Bunken Insatsusha (International Academic Printing Co., Ltd.), 270, 3-chome Totsuka-cho, Shinjuku-ku, Tokyo 160, Japan

* C. R. DePrima California Institute of Technology, Pasadena, CA 91109, will replace J. Dugundji until August 1974. 


\section{Pacific Journal of Mathematics}

Vol. 50, No. $2 \quad$ October, 1974

Mustafa Agah Akcoglu, John Philip Huneke and Hermann Rost, A counter example to the Blum Hanson theorem in general spaces .............

Huzihiro Araki, Some properties of modular conjugation operator of von

Neumann algebras and a non-commutative Radon-Nikodym theorem

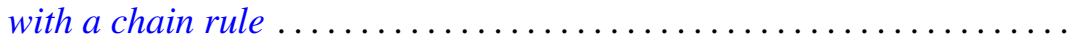

E. F. Beckenbach, Fook H. Eng and Richard Edward Tafel, Global properties of rational and logarithmico-rational minimal surfaces .....

David W. Boyd, A new class of infinite sphere packings ............. 383

K. G. Choo, Whitehead Groups of twisted free associative algebras ........

Charles Kam-Tai Chui and Milton N. Parnes, Limit sets of power series

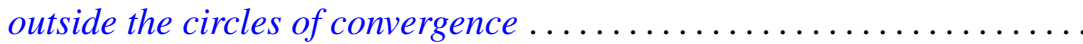

Allan Clark and John Harwood Ewing, The realization of polynomial

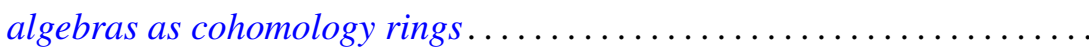

Dennis Garbanati, Classes of circulants over the p-adic and rational

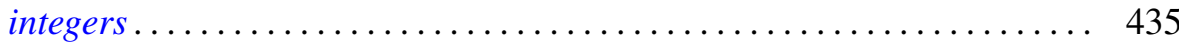

Arjun K. Gupta, On a "square" functional equation ................... 449

David James Hallenbeck and Thomas Harold MacGregor, Subordination and extreme-point theory ............................. 455

Douglas Harris, The local compactness of $v X \ldots \ldots . . . . . . . . . . . .4469$

William Emery Haver, Monotone mappings of a two-disk onto itself which fix the disk's boundary can be canonically approximated by homeomorphisms .................................. 477

Norman Peter Herzberg, On a problem of Hurwitz .................. 485

Chin-Shui Hsu, A class of Abelian groups closed under direct limits and subgroups formation ............................... 495

Bjarni Jónsson and Thomas Paul Whaley, Congruence relations and multiplicity types of algebras.....................

Lowell Duane Loveland, Vertically countable spheres and their wild sets.

Nimrod Megiddo, Kernels of compound games with simple components ....

Russell L. Merris, An identity for matrix functions ........ . .

E. O. Milton, Fourier transforms of odd and even tempered distributions ...

Dix Hayes Pettey, One-one-mappings onto locally connected generalized continua

Mark Bernard Ramras, Orders with finite global dimension

Doron Ravdin, Various types of local homogeneity. .

George Michael Reed, On metrizability of complete Moore spaces ...

Charles Small, Normal bases for quadratic extensions ..

Philip C. Tonne, Polynomials and Hausdorff matrices.... . . 